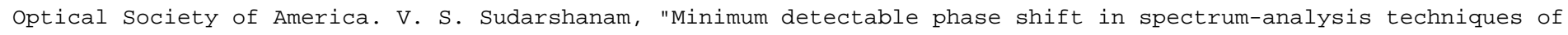
optical interferometric vibration detection," Appl. Opt. 31, 5997-6002 (1992). doi: 10.1364/AO.31.005997

\title{
Minimum detectable phase shift in spectrum-analysis techniques of optical interferometric vibration detection
}

\author{
V. S. Sudarshanam
}

\begin{abstract}
The minimum detectable phase shift indicated in recent experimental reports of new linear spectrumanalysis techniques of optical interferometric vibration detection is established as the direct consequence of the $1 / f$ noise voltage in the system components. The dynamic range and inaccuracy predicted by the simple theoretical model presented is in good agreement with experimental measurements. The conclusions of the analysis are compared with experimental reports of heterodyne shot-noise-limited optical systems. With this effective tool the generic class of spectrum-analysis techniques can be analyzed and relatively weighed to assess the effect of noise. This analysis is applicable to optical interferometry in general, although the experiments specifically involved fiber-optic modulators.
\end{abstract}

\begin{abstract}
Introduction
Optical interferometric methods have been widely used for the detection of vibration and dynamic displacement, both in noncontact and invasive configurations. ${ }^{1-6}$ Noncontact methods are generally implemented with the use of a laser and conventional optical elements such as beam splitters and lenses. Optical fibers have been incorporated to serve as simple light-transmission links to provide a flexibility of arrangement. Alternatively, the fiber itself could be utilized to measure the vibration through the stress-optic effect. In such an invasive technique the optical fiber is bonded onto the structure whose vibration is to be measured. ${ }^{7}$ For both the noncontact and invasive methods, the interferometer could be of the Mach-Zehnder, Michelson, or Fabry-Perot arrangements. This wide class of interferometric vibration-measurement methods has a common limitation in that the output intensity is a cosinusoidal function of the phase shift induced by the vibration.., 6 Hence a direct linear measurement of the phase shift, and therefore the vibration, is generally not possible. This is particularly true if the amplitude of vibration is considerably large. As fluctuations in ambient
\end{abstract}

The author is with the Fiber and Electro Optics Research Center, Bradley Department of Electrical Engineering, Virginia Polytechnic Institute and State University, Blacksburg, Virginia 24061-0111.

Received 5 December 1991.

0003-6935/92/285997-06\$05.00/0.

(C) 1992 Optical Society of America. temperature and pressure randomly affect the path difference between the two beams of the interferometer, the detected signal undergoes fading. ${ }^{8}$ This process results in randomly fluctuating intensity amplitudes of the vibration harmonics. As the phase shift is detected through an instantaneous intensity measurement, changes in the source intensity and frequency and the polarization states of the interfering beams also cause unpredictable variations.

Several demodulation schemes have been devised for dynamic phase detection in both conventional and fiber-optic interferometry. The heterodyne $e^{3,4}$ and active homodyne ${ }^{9}$ approaches offer attractive ways to eliminate signal fading. Nevertheless, passive homodyne techniques are simpler in that the problems associated with the Bragg cell in the heterodyne approach and the electronic reset glitch in the activefeedback approach do not exist. ${ }^{5}$ Spectrum-analysis techniques form a generic class of passive phasedetection schemes that is particularly suited to simple harmonic vibration..$^{1-8}$ Conventional spectrum-analysis methods consist of the $J_{1}(\max ), J_{n}$ (null), $J_{1} / J_{2}$, $J_{1} / J_{3}$, and the Bessel recurrence relation methods. ${ }^{3}$ These methods require the interferometer phase difference to be biased at $90^{\circ}$, which is the quadrature point about which the intensity is linear with the phase.

A recent report $t^{4}$ utilized the asymptotic relationship, $J_{n}^{2}(x)+J_{n+1}^{2}(x)=2 / \pi x$, between the Bessel functions of the first kind to determine the amplitude $x$ of the dynamic phase shift. This method involves measuring the photodetector voltage amplitude at 
the heterodyne carrier frequency with and without vibration and that of the first-order spectra. In comparison, an elegant technique named the $J_{1} \ldots J_{4}$ method that provided a self-consistent direct linear phase readout in a no-feedback, no-phase bias condition was reported. ${ }^{5}$ The phase measurement was unaffected by random fluctuations in the phase difference, the polarization states, or the source intensity. The need for such a linear technique arises in multimode-fiber interferometric sensor systems that are prone to intensity fluctuations. ${ }^{7}$ The $J_{1} \ldots J_{4}$ method was further modified in another report ${ }^{10}$ to extend its usefulness without disturbing the basic Bessel recurrence relationship used.

Although the two implementations involved different sets of optical and electronic elements, the behavior of the phase measurement as a function of the drive voltage to the fiber-optic phase-modulator test element was essentially the same. Whereas the mathematical expressions used to derive the phase shift from the Bessel relations were exact and did not impose any lower limit as such, practical measurements were limited to a minimum detectable phase shift (MDPS) of $0.1 \mathrm{rad}$ in both cases. Utilizing a phase modulator to upshift the contribution of the $J_{0}$ component to non-dc frequencies, I found that the $J_{0} \ldots J_{2}$ method $^{8}$ led to an improvement by an order of magnitude for the MDPS. This paper develops a simple theoretical model that establishes the observed lower bound as the direct consequence of the $1 / f$ noise voltage seen at the output. In excellent agreement with earlier experimental reports, the theoretical model also confirms the improvement in the MDPS for the $J_{0} \ldots J_{2}$ method and predicts the observed large error as a signal phase shift of $2.4 \mathrm{rad}$ is reached. With this simple tool the generic class of spectrum-analysis techniques can be relatively compared to determine the dynamic range and accuracy of phase detection in the presence of noise. It should be emphasized that this paper addresses optical interferometry in general, although the experiments specifically involved fiber phase sensors and modulators.

\section{Experimental Reports and $1 / f$ Noise Voltage}

A Mach-Zehnder homodyne interferometer formed from two single-mode fibers is considered here as an example. ${ }^{5}$ The light from a $\mathrm{He}-\mathrm{Ne}$ laser is split, guided within the fibers, and recombined to form a fringe pattern. An in-line fiber phase modulator is configured by bonding a bare section of the fiber onto a piezofilm. The piezofilm is driven by a sinusoidal excitation of amplitude $x$ at angular frequency $\omega$. It is this amplitude $x$ that is to be measured by a photodetector accessing the fringe pattern. The detector output is amplified and fed to a signal analyzer. The instantaneous voltage $V(t)$ can be written as $^{5,6}$

$$
\begin{aligned}
V(t)= & A+B\left[\left(J_{0}(x)-2 J_{2}(x) \cos 2 \omega t\right.\right. \\
& \left.+2 J_{4}(x) \cos 4 \omega t\right) \cos \phi_{0}(t)-\left(2 J_{1}(x)\right. \\
& \left.\left.\times \cos \omega t-2 J_{3} \cos 3 \omega t\right) \sin \phi_{0}(t)\right],
\end{aligned}
$$

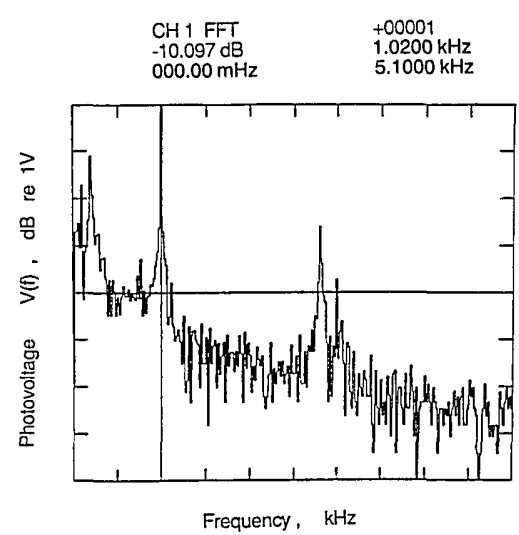

Fig. 1. Frequency spectrum of $V(t)$ for an input voltage of 57 $\mathrm{mV}_{\text {rms }}$ at $1.02 \mathrm{kHz}$ to the piezofilm. Analyzer bandwidth is 20 $\mathrm{Hz}$. CH1 FFT, fast Fourier transform of channel 1.

where $A=2 v, B=2 v b, v$ is the photovoltage from the fiber output assumed equal for both the fibers, $b$ is the fringe contrast or visibility, $J_{n}(x)$ are Bessel functions of the first kind, and $\phi_{0}(t)$ is the random phase drift caused by fluctuations in ambient temperature and pressure.

The signal analyzer converts $V(t)$ into an amplitudefrequency spectrum $V(f)$. Figure 1 shows a plot of $V(f)$ in decibels for a voltage of $57 \mathrm{mV}_{\text {rms }}$ applied to the piezofilm at $1.02 \mathrm{kHz}$ and an analyzer bandwidth of $20 \mathrm{~Hz}$. The peaks at the fundamental and odd harmonics alone are visible because for this plot the dc phase bias between the interferometer arms was adjusted to $\sim 90^{\circ}$, which is the quadrature point. The voltage noise floor is determined to vary as $1 / f$, with peaks at regular intervals corresponding to the line frequency of $50 \mathrm{~Hz}$. A similar $1 / f$ noise-voltage spectrum, or equivalently $1 / f^{2}$ power spectrum, has been reported for similar systems by several researchers. ${ }^{911,12}$ The main sources for this noise are the semiconductor junctions in the photodetector and the electronic circuitry following it. It is well known that the generation-recombination noise in semiconductors bears a $1 / f^{2}$ power spectrum..$^{13}$ However, it has also been shown that different samples of lasers exhibit varying levels of $1 / f^{2}$ noise power, with care taken to avoid any ambiguities in the measurement. ${ }^{11}$ Thus the laser source also contributes to the $1 / f$ noise voltage. $^{14}$ For values of $x$ below $2.6 \mathrm{rad}$, where $J_{1}(x)=J_{2}(x)$, the value of the Bessel function falls off with higher orders. Hence, for low values of $x, V_{3}$ and $V_{4}$ would be closer to the noise floor at their respective frequencies as compared with $V_{1}$ and $V_{2}$. Here the $V_{i}$ 's stand for the voltage amplitude of the $i$ th frequency component in Eq. (1). As the $J_{1} \ldots J_{4}$ method utilizes the components $V_{1}$ to $V_{4}$, the error introduced by the noise floor gets translated into inaccuracy in the measurement of $x$, resulting in a MDPS. 


\section{Theoretical Model}

Based on the Bessel recurrence relation, the $J_{1} \ldots J_{4}$ method uses the following expression for measuring $x$ :

$$
x^{2}=24 V_{2} V_{3} /\left(V_{1}+V_{3}\right)\left(V_{2}+V_{4}\right),
$$

where $V_{i}=2 B g J_{i}(x), g=\cos \phi_{0}$ (or $\sin \phi_{0}$ ) for $i$ even (or odd). As a result of the $1 / f$ noise voltage, the measured value $x^{\prime}$ would deviate from the expected value $x$ according to

$$
\begin{aligned}
\left(x^{\prime}\right)^{2}= & 24\left(J_{2}(x)+K / 2\right)\left(J_{3}(x)+K / 3\right) /\left(J_{1}(x)\right. \\
& \left.+J_{3}(x)+1.33 K\right)\left(J_{2}(x)+J_{4}(x)+0.75 K\right),
\end{aligned}
$$

where $K=\Delta V_{1} / 2 B$ and $\Delta V_{1}$ is the noise voltage at the fundamental frequency. The value of $K$ can be determined accurately from the spectrum in Fig. 1. The average phase-shifting coefficient ${ }^{5}$ was determined from the $J_{1}(\max ), J_{1} \ldots J_{4}, J_{0} \ldots J_{2}, J_{0}$ (null), and ramp-input methods. The input voltage of 57 $\mathrm{mV}_{\mathrm{rms}}$ corresponded to an $x$ of $0.13 \mathrm{rad}$. With this value, the ratio of signal and noise voltages at 1.02 $\mathrm{kHz}$ in Fig. 1, and $\phi_{0}=90^{\circ}, K$ was determined as 0.000587 .

Figure 2 shows a plot of $x^{\prime}$ and the error $\Delta x=$ $\left(x^{\prime}-x\right)$ using Eq. (3) as a function of $x$. The secondorder least-squares fit for the experimental plot of phase shift versus input voltage in Fig. 2 of Ref. 5 was determined to be $y=\left(2.66 x-0.078 x^{2}-0.317\right)$ for phase shifts above 0.5 rad. The intercept of -0.317 rad was specific to the piezofilm configuration used, whereas in general such an intercept may not be seen for all modulators. Therefore, the intercept was corrected for and the best fit was utilized to replot the experimental points on the changed axes of the measured phase versus the expected phase, as shown in Fig. 2 here. The MDPS is defined as the value of $x$ for which $\Delta x=x$, or equivalently the point at which

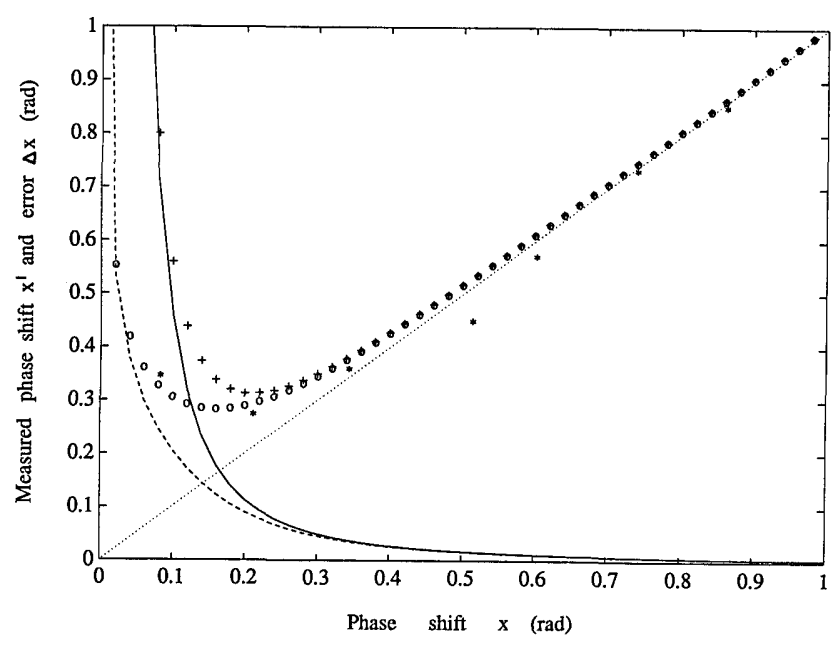

Fig. 2. Measured phase shift $x^{\prime}$ and the error $\Delta x=\left(x^{\prime}-x\right)$ as a function of the expected phase shift $x$. Dashed curve, $\Delta x$; solid curve, $\mathrm{d} x$; dotted curve; $\Delta x=x$; asterisks, experimental values; open circles, $x^{\prime}=x+\Delta x$; crosses, $x^{\prime}=x+\mathrm{d} x$. the plot of $\Delta x$ versus $x$ intersects the straight line $\Delta x=x$ (see Fig. 2). Thus an MDPS of $0.13 \mathrm{rad}$ is seen for $K=0.000587$, which is in good agreement with the experimentally measured values. It may be worthwhile to find the relative magnitude of $\Delta x$ with respect to $x$. If $\Delta x$ was considered to be small, the derivative $\mathrm{d} x$ could replace $\Delta x$; the plots of $x^{\prime}=x+\mathrm{d} x$ and $\mathrm{d} x$ as a function of $x$ are also shown in Fig. 2 . It can be seen that the approximation $\Delta x=d x$ leads to a large discrepancy between experimental and expected values when $x$ is below 0.2 rad. Hence Eq. (3) provides the correct model for the observed experimental results.

\section{Analysis}

With Eq. (3) as its basis, Fig. 3 shows the error $\Delta x$ as a function of $x$ for three different values of $K$. The standard deviation of the phase measured over 50 trials for each point in the experimental plot of Ref. 5 did not exceed $\pm 3 \mathrm{mrad}$. From Fig. 3 one sees a dynamic range of approximately 1 to $4 \mathrm{rad}$ within that accuracy, which is in good agreement with Ref. 5 . A larger dynamic range is possible with a lesser $K$. The $K$ would be lesser for a higher $\omega$, e.g., in going from $1.02 \mathrm{kHz}$ as in Fig. 1 to $30 \mathrm{kHz}, K$ would decrease by $30 \mathrm{~dB}$. Alternatively, a 10-dB increase in the difference between the signal and the noise voltage at $1.02 \mathrm{kHz}$ decreases $K$ to $1 / 5469$ and thereby increases the dynamic range by $\sim 2 \mathrm{rad}$ for an accuracy of \pm 3 mrad. It has been shown ${ }^{15}$ that such a reduction in the noise floor is in fact possible with the use of two detectors shifted in the optical phase by $180^{\circ}$.

The effect of random phase drift on the measurement of $x$ is now studied. It is seen from Eqs. (1) and (2) that if $V_{2}$ and $V_{4}$ are zero at $\phi_{0}=90^{\circ}, V_{1}$ and $V_{3}$ would be zero at $\phi_{0}=0^{\circ}$. Therefore it is necessary to avoid measurements near $\phi_{0}=n \pi / 2$, where $n$ is an integer. As $\phi_{0}$ moves away from these points the

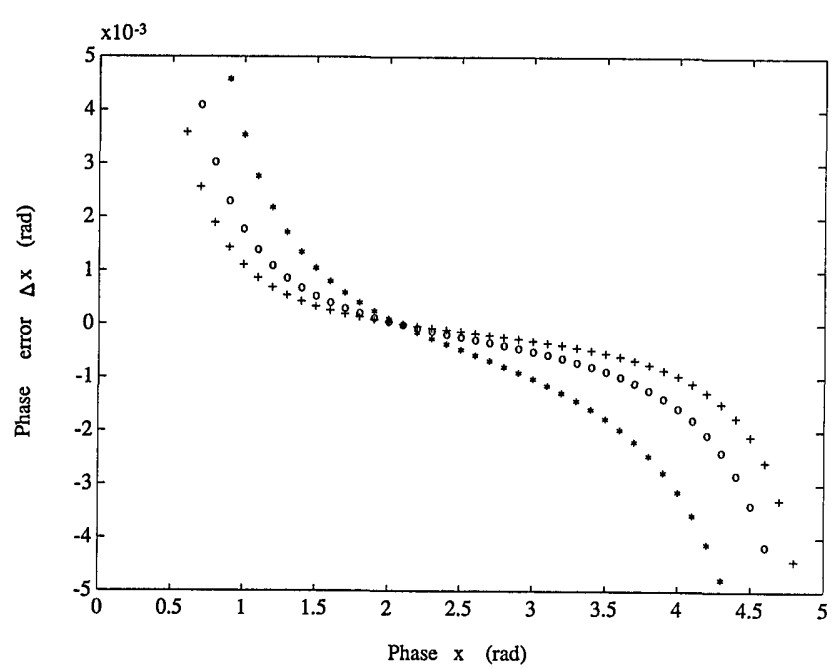

Fig. 3. Error $\Delta x$ in the measured phase shift as a function of the phase shift $x$ for different values of $K$. Asterisks, $K=1 / 1701$; open circles, $K=1 / 3400$; crosses, $K=1 / 5469$. 
accuracy of the measurement becomes higher. To see this phenomenon one needs to write the measured phase $x^{\prime}$ as a function of $\phi_{0}$ for a given $x$ :

$$
\begin{aligned}
\left(x^{\prime}\right)^{2}= & 24\left(Q J_{2}+K / 2\right)\left(P J_{3}+K / 3\right) /\left[\left(J_{1}+J_{3}\right) P\right. \\
& +1.33 K]\left[\left(J_{2}+J_{4}\right) Q+0.75 K\right]
\end{aligned}
$$

where $P=\sin \phi_{0}$ and $Q=\cos \phi_{0}$. Figure 4 shows the measured phase $x^{\prime}$ as a function of $\phi_{0}$ for $x=2.405$ rad for three values of $K$. It is seen that (a) for $K=$ 0 , the measurement of $x$ by the $J_{1} \ldots J_{4}$ method is independent of $\phi_{0}$ as indicated by the ideal Eq. (2), and (b) the range of $\phi_{0}$ over which the error is below a certain limit, say $\pm 3 \mathrm{mrad}$, is larger for a lesser $K$ and is dependent on the value of $x$.

The $J_{0} \ldots J_{2}$ method $^{8}$ is now analyzed with the model tested above. Basically this method consists in superimposing the signal phase shift of $M \mathrm{rad}$, which is to be measured, on a sinusoidal reference phase modulation of $L$ rad at a higher frequency. The following experimental observations are verified specifically: (a) the MDPS of $0.01 \mathrm{rad}$ and (b) the larger error near $M=2.4 \mathrm{rad}$. The measured phase shift $M^{\prime}$ for the $J_{0} \ldots J_{2}$ scheme can be written as (see Ref. 8 for ideal $M$ )

$$
M^{\prime}=2\left(G^{\prime} H^{\prime} I^{\prime}\right)^{1 / 2} J^{\prime-1 / 2} /\left(G^{\prime}+T^{\prime}\right),
$$

where

$$
\begin{aligned}
G^{\prime} & =2 B P J_{1}(L) J_{0}(M)+K / 2, \\
H^{\prime} & =2 B Q J_{1}(L) J_{1}(M)+K / 2.4, \\
I^{\prime} & =2 B P J_{2}(L) J_{1}(M)+K / 4.4, \\
J^{\prime} & =2 B Q J_{2}(L) J_{0}(M)+K / 4, \\
T^{\prime} & =2 B P J_{1}(L) J_{2}(M)+K / 2.8,
\end{aligned}
$$

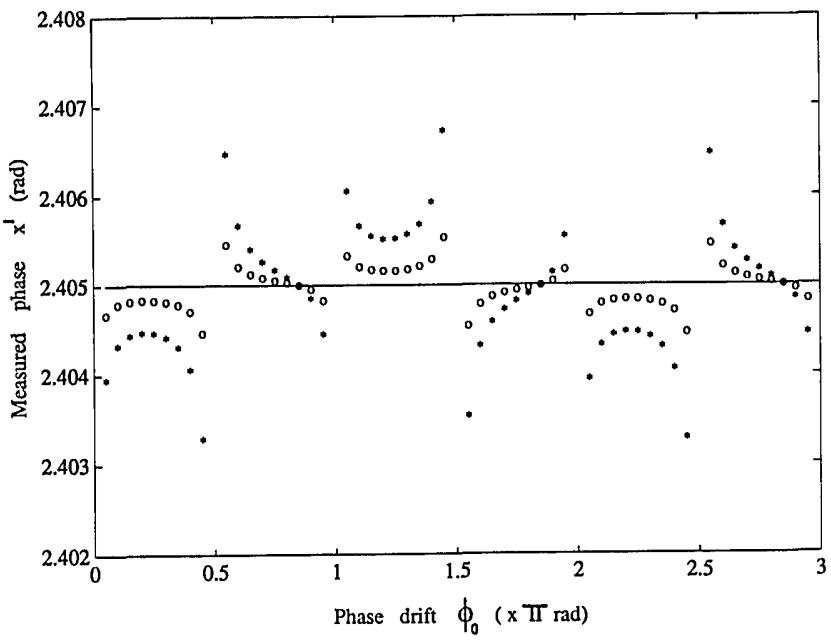

Fig. 4. Measured phase shift $x^{\prime}$ as a function of the random phase shift $\phi_{0}$ for $x=2.405 \mathrm{rad}$. The horizontal line at $x^{\prime}=2.405 \mathrm{rad}$ corresponds to $K=0$. Asterisks, $K=1 / 1701$; open circles, $K=$ $1 / 5469$. Gaps in the plots indicate discontinuities whose values have not been plotted here.
The numerals dividing $K$ in Eqs. 6(a)-6(e) are based on the signal modulation frequency of $400 \mathrm{~Hz}$ and reference modulation frequency of $2 \mathrm{kHz}$, with $K$ measured at $1.02 \mathrm{kHz}$ as described earlier.

In the following discussion it is assumed that $P=$ $Q=\cos (\pi / 4)$. This assumption is based on the insensitivity of the phase measurement proved earlier, within a certain inaccuracy, with respect to variations in $\phi_{0}$. It has been verified that the MDPS, as defined earlier, is lower for higher $K$ for any value of $L$. Figure 5 shows a plot of the signal phase-shift error $\Delta M$ as a function of the reference phase shift $L$ for different values of the signal phase shift $M$ with $K=$ $1 / 1701$. It is seen that for the value of $L=1 \mathrm{rad}$ chosen for the experiments in Ref. 8, a range of $M$ from approximately 0.01 to 2 is measurable within an error of $\sim 2$ mrad. The experimentally observed inaccuracy was $\pm 1 \mathrm{mrad}$ for most of this range. As stated in Ref 8 , Fig. 5 shows that $M$ can be measured to within an accuracy of $1 \mathrm{mrad}$ independent of the value of $L$, which can be chosen over a wide range. The experimental fact that the error $\Delta M$ becomes large as the value of $M$ reaches $2.4 \mathrm{rad}$ is seen from Fig. 6. This phenomenon happens because $J_{0}(M)$ would approach zero closer as $M$ crosses $2 \mathrm{rad}$. In good agreement with the experimental value of \pm 1 mrad for the inaccuracy, Fig. 6 shows an error of similar magnitude for $L=1.5 \mathrm{rad}$ over the range of $M$ of interest. However, the experimental value of 10 mrad for the MDPS was far higher than the $1 \mathrm{mrad}$ predicted by Fig. 6 . This higher value was to be expected because the function generator driving the piezofilm had a $2 \%$ instability for each range. Thus, for the lowest range of voltage of $0.2 V_{\text {rms }}$ used in Ref. 8 , the instability in the phase shift turns out to be 9 mrad. It should be noted that the $J_{0} \ldots J_{2}$ method was relatively insensitive to the error caused by random phase drifts away from the points of discontinuity.

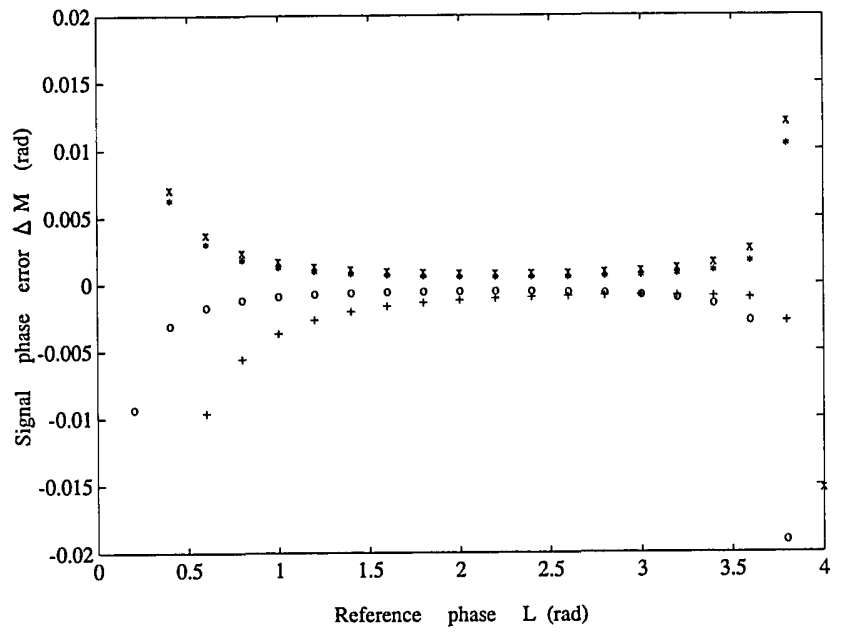

Fig. 5. Error $\Delta M$ in the signal phase shift measured by the $J_{0} \ldots$ $J_{2}$ method as a function of the reference phase shift $L$, for different values of signal phase shift $M ; K=1 / 1701$. Exes, $M=0.01 \mathrm{rad}$; asterisks, $M=0.3 \mathrm{rad}$; open circles, $M=1.5 \mathrm{rad}$; crosses, $M=2$ rad. 


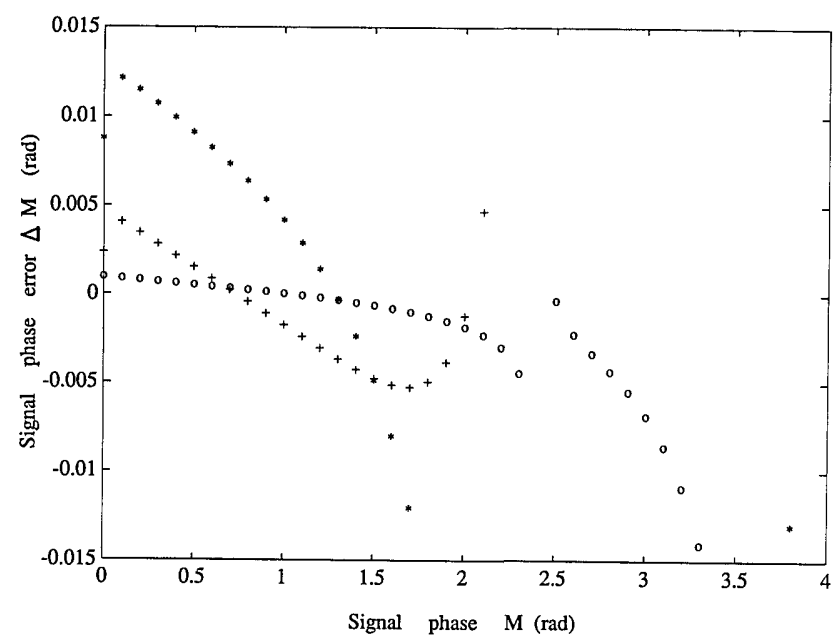

Fig. 6. Error $\Delta M$ in the signal phase shift measured by the $J_{0} \ldots$ $J_{2}$ method as a function of the signal phase shift $M$, for different values of reference phase shift $L ; K=1 / 1701$. Asterisks, $L=0.3$ rad; open circles, $L=1.5 \mathrm{rad}$; crosses, $L=3.7 \mathrm{rad}$.

The analysis presented above explains all the experimentally observed characteristics of the $J_{1} \ldots J_{4}$ and the $J_{0} \ldots J_{2}$ methods. It should be pointed out that several expressions similar to Eqs. (2) and (5) could be utilized to determine the dynamic phase change. The noise model presented here predicts the MDPS and the accuracy for each such technique and permits a judicious choice of the expression to be used for a given application.

\section{Comparison with Heterodyne Shot-Noise-Limited Systems}

It would be worthwhile to compare the MDPS achievable from the homodyne $J_{1} \ldots J_{4}$ method with that of a heterodyne shot-noise-limited system. In a heterodyne interferometer, where the optical frequency in one of the arms is shifted by $\Omega$, the instantaneous voltage $V(t)$ can be written in a like manner to Eq. (1):

$$
V(t)=A+B \sum_{n=-\infty}^{\infty} J_{n}(x) \cos \left[(\Omega-n \omega) t+\phi_{0}(t)\right] .
$$

It is seen from Eq. (7) that the random phase term $\phi_{0}(t)$, which now appears as the phase constant of each $V_{i}(f)$, would not affect the measurement of $x$ if one utilizes the digital Fourier transform of $V(t)$ and reads only the magnitude of the voltage $V_{i}(f)$ at each frequency. It should be pointed out that the approximation $J_{n}^{2}+J_{n+1}^{2}=2 /(\pi x)$ utilized in the heterodyne system of Ref. 4 holds good only for large values of $x$. Moreover, the application of the noise analysis presented here in the earlier sections to this expression has shown that the derivation of $x$ from such a relation results in an oscillatory error and is therefore not considered here. For comparison of the MDPS here, assume that the signal phase shift $x$ is very small, $x \ll 1$, so that the following approximation for the Bessel function can be used:

$$
J_{n}(x) \cong x^{n} /\left(2^{n} / n !\right)
$$

The phase shift $x$ can then be derived by (a) considering $^{3}$ either of the first sideband components at $\Omega+\omega$ or $\Omega-\omega$ frequencies, both of which would be proportional to $x$, or (b) considering ${ }^{16}$ the power ratio of the first sideband, $P_{1}$, and the carrier frequency, $P_{0}$, using the relation

$$
P_{1} / P_{0}=\left[J_{1}(x) / J_{0}(x)\right]^{2}=[x / 2]^{2} .
$$

For a heterodyne Mach-Zehnder shot-noise-limited interferometer, the signal photocurrent $i_{s}$ and the shot-noise current $i_{n}$ can be written as ${ }^{3}$

$$
\begin{aligned}
i_{s} & =x b I /(2)^{1 / 2}, \\
i_{n} & =(4 e I B)^{1 / 2},
\end{aligned}
$$

where $l$ is the photocurrent from each of the interfering beams, $e$ is the electronic charge, and $B$ is the detection bandwidth. The MDPS is obtained by equating Eqs. (10) and (11) for a signal-to-noise ratio (SNR) of 1 , and assuming $b=1$, as

$$
\begin{aligned}
\mathrm{MDPS} & =(8 e B / I)^{1 / 2}, \\
& =4\left(h \nu B / \eta P_{0}\right)^{1 / 2},
\end{aligned}
$$

where $\nu$ is the unshifted optical frequency, $h$ is the Planck's constant, $\eta$ is the detector quantum efficiency, and $P_{0}$ is the laser power. In comparison with a homodyne Michelson interferometer, the factor 4 in Eq. (12) arises because (a) the Mach-Zehnder configuration has half the sensitivity of the Michelson one, and (b) the heterodyne method utilizes only one of the sidebands. ${ }^{3}$ Substituting the typical numerical values ${ }^{16}$ for the photomultiplier detection of $P_{0}=$ $17 \mathrm{nW}, B=1 \mathrm{kHz}, \eta=5 \%$, and $h \nu=3.1 \times 10^{-19} \mathrm{Ws}$, we see that the MDPS becomes 2.41 millirad. It is to be emphasized that this MDPS holds only when the $J_{1}$ component alone is considered. The reason the MDPS $(0.1 \mathrm{rad})$ for the homodyne $J_{1} \ldots J_{4}$ method is much higher than this value is that whereas the noise has a $1 / f$ behavior, the Bessel function magnitude at successive frequencies follows Eq. (8) so that the SNR for successive frequencies decreases rapidly for $x \ll$ 1. However, phase detection by considering only the $J_{1}$ component is susceptible to source-intensity changes and visibility fluctuations. It is in overcoming this serious problem through linearization by the $J_{1} \ldots J_{4}$ method that the MDPS is sacrificed.

From the experimental results of Ref. 16 we have, for a displacement of $24 \mathrm{~nm}$ corresponding to a phase shift of $0.336 \mathrm{rad}$, a shot-noise level of $0.23 \mathrm{~nm}$ and a SNR of $\sim 52.5 \mathrm{~dB}$ for the $J_{0}$ component. The corresponding value of $K$ determined from these values is 0.0023 . Utilizing this value and modifying the noise analysis presented here under the assumption that shot noise is the same for all frequencies, one can see a MDPS of $0.0045 \mathrm{rad}$ for the $J_{1} \ldots J_{0}$ method of Ref. 16. This is in excellent agreement with the value of $0.0032 \mathrm{rad}$ calculated directly from the observed noise floor level and $\beta=14 \mu \mathrm{m}^{-1}$ in Ref. 16 . In comparison, the MDPS for the $J_{1} \ldots J_{4}$ method for a 
heterodyne shot-noise-limited system can be found by extrapolating the plot of Fig. 1 to higher frequencies where the assumption of shot noise is valid. As the value of $K$ dcreases by $30 \mathrm{~dB}$ in going over to frequencies higher than $30 \mathrm{kHz}$, the MDPS is determined to be 0.065 rad. Thus the MDPS increases by more than an order of magnitude when one measures $x$ from four components instead of just one.

\section{Conclusion}

A simple yet powerful theoretical model based on the $1 / f$ noise voltage in system components has been presented to predict the minimum detectable phase shift, dynamic range, and accuracy of spectrumanalysis techniques of interferometric phase detection. The excellent agreement with experimental reports indicates the versatility of the analysis for predicting the noise behavior of, and choice of optimum values for reference phase modulation in, such spectrumanalysis techniques.

The author thanks R. O. Claus for constant encouragement. This research was partially supported by the Center for Innovative Technology, Virginia Polytechnic Institute and State University.

\section{References}

1. H. A. Deffarari, R. A. Darby, and F. A. Andrews, "Vibrational displacement and mode shape measurement by a laser interferometer," J. Acoust. Soc. Am. 42, 982-990 (1967).

2. B. J. Pernick, "Self-consistent and direct reading laser homodyne measurement technique," Appl. Opt. 12, 607-610 (1973).

3. Y. Ohtsuka, "Dynamic measurements of small displacements by laser interferometry," Trans. Inst. Meas. Control 4, 115124 (1982).

4. C. S. Vikram and T. E. McDevitt, "Simple spectrum analysis in laser Doppler studies of sinusoidal vibrations," Opt. Eng. 28, 922-925 (1989).
5. V. S. Sudarshanam and K. Srinivasan, "Linear readout of dynamic phase change in a fiber optic homodyne interferometer," Opt. Lett. 14, 140-142 (1989).

6. V. S. Sudarshanam and K. Srinivasan, "Universal dynamic phase calibration technique for fiber optic interferometric sensors and phase modulators," Opt. Lett. 14, 1287-1289 (1989).

7. V. S. Sudarshanam, "Multimode fiber axial strain sensor utilising end reflection interference," J. Mod. Opt. 39, 615624 (1992).

8. V. S. Sudarshanam, "New spectrum analysis technique for interferometric vibration measurement," Opt. Commun. 88, 291-294 (1992).

9. K. P. Koo and G. H. Sigel, Jr., "An electric field sensor utilising a piezoelectric polyvinylidene fluoride (PVF2) film in a single mode fiber interferometer," IEEE J. Quantum Electron. QE-18, 670-675 (1982).

10. W. Jin, L. M. Zhang, D. Uttamchandani, and B. Culshaw, "Modified $J_{1} \ldots J_{4}$ method for linear readout of dynamic phase changes in a fiber optic interferometer," in Fiber Optic Sensors IV, R. T. Kersten, Proc. Soc. Photo-Opt. Instrum. Eng. 1267, 153-157 (1990).

11. J. H. Cole, "Low frequency laser noise of several commercial lasers," Appl. Opt. 19, 1023-1025 (1980).

12. A. Dandridge, A. B. Tveten, R. O. Miles, and T. G. Giallorenzi, "Laser noise in fiber optic interferometer systems," Appl. Phys. Lett. 37, 526-528 (1980).

13. F. N. Hooge, T. G. M. Kleinpenning, and L. K. J. Vandamme, "Experimental studies of $1 / f$ noise," Rep. Prog. Phys. 44, 480-532 (1981).

14. O. Ishida, "Delayed self heterodyne measurement of laser frequency fluctuations," IEEE J. Lightwave Technol. 9, 15281533 (1991).

15. A. Dandridge, "Noise reduction in fiber optic interferometer systems," Appl. Opt. 20, 2337-2339 (1981).

16. R. Daendliker and J.-F. Willemin, "Measuring microvibrations by heterodyne speckle interferometry," Opt. Lett. 6, 165-167 (1981). 